\title{
The effects of founding bottlenecks on genetic variation in the European starling (Sturnus vulgaris) in North America
}

\author{
PAUL R. CABE \\ Department of Ecology, Evolution, and Behaviour, University of Minnesota, St. Paul, MN 55108, U.S.A.
}

\begin{abstract}
Genetic variation in European starlings in North America was examined using enzyme electrophoresis and compared to that in their home range. The effect of the founding bottleneck matched theoretical predictions. Heterozygosity was unaffected, whereas allelic diversity may have decreased. Results from this study and others suggest that theoretical predictions of bottlenecks are robust for allozyme data, and applicable under a wide variety of conditions.
\end{abstract}

Keywords: European starling, introduced species, population bottlenecks.

\section{Introduction}

The concepts of population bottlenecks and founder effects have a long history in evolutionary genetics. The microevolutionary effects of population bottlenecks on the genetic variation within populations have received much attention, both in theoretical treatments and empirical studies. Many empirical studies have examined the genetic variation within populations known or presumed to have undergone recent bottlenecks, and others have attempted to relate observed levels of genetic variation to hypothesized bottlenecks in the past (O'Brien et al., 1987; Packer et al., 1991; Wayne et al., 1991). Although theoretical treatments (Nei et al., 1975; Chakraborty \& Nei, 1977; Sirkkomma, 1983; Watterson, 1984; Maruyama \& Fuerst, 1985) have allowed predictions about the loss of genetic variation resulting from bottlenecks, these predictions have rarely been tested explicitly (but see Leberg, 1992). These predictions would require accurate information about the population from which founders were drawn, the size of the population during the bottleneck, the length of time during which the population was small, and the growth rate of the population following the bottleneck. Unfortunately, these data are lacking for most cases.

Understanding the effects of bottlenecks on populations is important for several reasons. They may be expected to leave their signature on populations;

Current address: Biology Department, St. Olaf College, Northfield, MN 55057 U.S.A. E-mail: cabe@stolaf.edu interpreting this signature may aid in reconstructing population histories. For example, O'Brien et al. (1987) interpreted a lack of allelic variation as evidence for several severe population bottlenecks at some time in the recent past. In addition, as many more populations dwindle in size as a result of human activities, it is important to understand the loss of genetic variation resulting from these demographic changes.

The European starling (Sturnus vulgaris) in North America is a good candidate to test the theoretical predictions of bottlenecks. All starlings in North America are thought to be derived from introductions made in 1890 and 1891 to Central Park, New York (see Cabe, 1989). Although there is some disagreement on the precise number, it is believed that about 60 were released in 1890, and 40 more in 1891 (see Cabe, 1993). Although the source of the founders is no longer known, it was likely to have been Great Britain; Eugene Schiefflin, who arranged the release, was attempting to import and establish all of the birds mentioned in Shakespeare's writings. The exact source is not crucial given the population structure of this species in Europe. In Evans's (1980, p. 2) opinion, 'continental Western Europe may be regarded effectively as a single panmictic population.'

Because of the starling's visibility, and the interest of professional and amateur ornithologists, its status and range subsequent to introduction are reasonably well documented. The starling's life history is well known (Feare, 1984; Cabe, 1993), making estimates of relevant population parameters possible. 
The genetic structure of starlings based on allozyme variation has been characterized for their native range in Great Britain (Evans, 1980; Ross, 1983) and for introduced populations in New Zealand (Ross, 1983). This allows the direct comparisons reported here between the presumed source population, other introduced populations, and North American populations.

\section{Methods}

Starlings were sampled from four widely separated populations in early summer to obtain individuals that bred locally. A large sample $(n=78)$ was collected in June and July 1987 and 1988 from feeding areas in Augusta Co., VA. Smaller samples were collected from Bennington Co., VT $(n=19)$, Eagle Co., CO $(n=22)$ and Shasta Co., CA $(n=23)$. Tissue samples (liver, heart, muscle, blood) were taken immediately, and stored in cryovials in liquid $\mathrm{N}_{2}$.

Genetic variation was examined using starch gel electrophoresis. In order to compare results with those published for starlings in their native range, the electrophoretic procedures in general replicated those of Ross (1983).

Tissue samples were homogenized in a phenoxyethanol extraction solution (1.5\% 2-phenoxyethanol, $0.25 \mathrm{~m}$ sucrose, $0.1 \mathrm{~m} \mathrm{pH} 7.5$ phosphate buffer); this extraction procedure differs slightly from the Ross (1983) protocol. The extract was centrifuged, and the supernatant absorbed onto wicks cut from Whatman no. 1 filter paper or stored in liquid nitrogen when not in use. Gels were made with $11.5 \%$ Sigma electrostarch and run at $5^{\circ} \mathrm{C}$.

Three citrate-amine buffer systems were used: (i) $\mathrm{pH} 6.1,0.004 \mathrm{~m}$ citric acid with the addition of $\mathrm{N}$-(3-aminopropyl)-morpholine; (ii) $\mathrm{pH} 7.5$, prepared from the $\mathrm{pH} 6.1$ buffer with the addition of 1,3-bis(dimethylamino)-2-propanol to adjust $\mathrm{pH}$; (iii) $\mathrm{pH} 8.0,0.157 \mathrm{~m}$ citric acid adjusted to the desired $\mathrm{pH}$ with tris(hydroxymethyl)aminomethane. For the pH 6.1 and pH 7.5 systems, gel buffers were 5\% of the ionic strength of the electrode buffer; for the pH 8.0 system, the gel buffer was $3.3 \%$ of the electrode buffer.

Gels were sliced, and stained following Evans (1980) recipes using agar overlays. Minor adjustments included the following: (i) Est-1 and Est-2 used fast blue RR, and $2.5 \mathrm{~mL}$ of a solution of $1 \%$ substrate dissolved in acetone; (ii) both Pep- $A$ and $P e p-C$ showed good activity using leucyl-alanine as a substrate with $1 \mathrm{~mL}$ of $10 \%$ o-dianisidine replacing 3-amino-9-ethyl-carbazole. Additional details of electrophoretic procedure can be found in Cabe (1994).

Genotypes were inferred from electrophoretic phenotypes. The variable loci examined, with the exception of Pep- $A$ and Pep-C, have been checked for Mendelian inheritance (Evans, 1980; Romagnano, 1987). The interpretation of the Est-2 locus was problematic; variable activity necessitated multiple gel runs, and even so, some individuals were not scored.

In order to identify electrophoretic variants accurately, all individuals which were heterozygous or homozygous for rare alleles were run multiple times, with individuals exhibiting questionable alleles run in adjacent lanes on the same gel. Common alleles were designated as 100 , and other alleles designated on the basis of their relative mobility. By using the same protocols as Ross (1983), every effort was made to identify comparable genetic variation.

Alleles from North American samples were named in concordance with those reported by Ross (1983) for starlings in Great Britain and New Zealand. For instance, Ross noted three rare alleles at the Pep- $A$ locus, two which migrated more slowly than the most common allele, and one which migrated faster. Using similar gel buffers, one slower allele was identified in this study. Its relative mobility was $94.6 \%$ of the most common allele, so this was considered to be equivalent to the 95 allele identified by Ross.

GENESYS software (Corbin \& Wilkie, 1988) was used for calculations of allelic frequencies, observed heterozygosities and expected heterozygosities (with corrections for sample size; Nei, 1978), $F_{\text {IS }}$ for each polymorphic locus, and $F_{\mathrm{ST}}$ values; calculation of $F$-statistics was based on Wright (1978).

\section{Results}

Fifteen enzyme systems representing 23 putative loci were examined in this study. Of these, 17 were monomorphic: Acp, $\alpha-G p d h-1$ and $\alpha-G p d h-2$ (two zones on the gel showed activity but neither varied), Idh-1, Idh-2, Ldh-1, Ldh-2, Mdh-1, Mdh-2, Me, Mpi, Pgdh, Pgi, Sdh, Sod-1, Sod-2 and Xdh. The six variable loci were Est-1, Est-2, Pep-A, Pep-C, Pgm-1 and $P g m-2$. In general, the enzyme variability detected is consistent with prior studies in North America and elsewhere (Evans, 1980; Ross, 1983; Romagnano, 1987). Difficulties with enzyme activity for Est-2 made scoring genotypes difficult and the data from this locus somewhat suspect; relevant statistical analyses were performed twice, including and excluding this locus.

(c) The Genetical Society of Great Britain, Heredity, 80, 519-525. 
The frequencies of rare alleles at the six variable loci pooled over all localities are given in Table 1. In all cases, these loci were reported as variable in Great Britain. In Great Britain, the average number of alleles per variable locus was 3.33 (Ross, 1983), whereas in North America only two alleles were detected at each polymorphic locus.

Heterozygosity was calculated from the genotypic frequency data with corrections for sample size (Nei, 1978). Based on the 23 loci surveyed the corrected heterozygosity is $0.0332 \pm 0.0101$ (uncorrected value $0.0324 \pm 0.0098)$. To be comparable to the values reported by Ross, heterozygosities were also calculated assuming 18 instead of 17 monomorphic loci. Heterozygosity calculated in this way is $0.0318 \pm 0.0097$ (uncorrected value $0.0311 \pm 0.0098$ ). If data from the Est-2 locus are excluded, the corrected heterozygosity measured in this study is 0.0304 (uncorrected value 0.0298); exclusion of this locus has little effect on this estimate. Unfortunately, the data presented by Ross (1983) do not allow the heterozygosity to be recalculated excluding this locus.

At each variable locus, the genotypic frequencies for each location were tested against the expectations of Hardy-Weinberg equilibrium using both the chi-squared test and the $G$-test. In only one case (Est-2, Colorado) did the observed genotypic frequencies differ significantly $(0.01<P<0.025)$ from zero. This single case (an apparent homozygote for the rare 105 allele) could alternatively represent a heterozygote for a null allele. An analysis of $F_{\text {IS }}$ and genotypic frequencies was performed to evaluate the assumption of random mating within populations. Values for $F_{\text {IS }}$ based on different loci ranged from $-0.0677 \pm 0.0669$ to $0.2416 \pm 0.2529$

Table 1 Allelic frequencies of uncommon alleles estimated from electrophoretic data for introduced European starlings (Sturnus vulgaris) in North America and New Zealand, and from their native range in Great Britain (data from New Zealand and Great Britain pooled over localities, from Ross, 1983; additional data from Great Britain from Evans, 1980)

\begin{tabular}{|c|c|c|c|c|c|c|c|c|}
\hline Locus & Allele & Virginia & Vermont & Colorado & California & $\begin{array}{l}\text { U.S.A. } \\
\text { pooled }\end{array}$ & $\begin{array}{c}\text { New } \\
\text { Zealand } \\
(N=299)\end{array}$ & $\begin{array}{c}\text { Great } \\
\text { Britain } \\
(N=298)\end{array}$ \\
\hline \multirow[t]{2}{*}{ Est-1 } & 107 & 0.0200 & - & 0.0500 & 0.0217 & $\begin{array}{c}0.0219 \\
(0.0405)^{1}\end{array}$ & - & 0.0017 \\
\hline & 117 & 0.0267 & 0.0526 & 0.0250 & 0.0435 & $\begin{array}{c}0.0219 \\
(0.0378)^{1}\end{array}$ & 0.0217 & 0.0067 \\
\hline \multirow[t]{2}{*}{ Est-2 } & 95 & - & - & - & - & - & 0.1582 & $\begin{array}{c}0.0684 \\
(0.022)^{2}\end{array}$ \\
\hline & 105 & 0.0424 & - & 0.1111 & 0.0455 & 0.0408 & 0.1400 & $\begin{array}{c}0.0516 \\
(0.031)^{2}\end{array}$ \\
\hline$I d h-2$ & 144 & - & - & - & - & - & 0.0318 & 0.0017 \\
\hline$L d h-1$ & 133 & - & - & - & - & - & 0.0060 & - \\
\hline \multirow[t]{3}{*}{$L d h-2$} & 57 & - & - & - & - & - & 0.0083 & - \\
\hline & 76 & - & - & - & - & - & - & 0.0017 \\
\hline & 181 & - & - & - & - & - & - & 0.0017 \\
\hline$M p i$ & 83 & - & - & - & - & - & - & 0.0050 \\
\hline \multirow[t]{3}{*}{ Pep- $A$} & 89 & - & - & - & - & - & - & 0.0017 \\
\hline & 95 & 0.0833 & 0.0789 & 0.0455 & 0.0682 & 0.0745 & 0.1129 & 0.1347 \\
\hline & 106 & - & - & - & - & - & - & 0.0067 \\
\hline \multirow{3}{*}{ Pep-C } & 82 & - & - & - & - & - & - & 0.0034 \\
\hline & 89 & - & - & - & - & - & - & 0.0017 \\
\hline & 107 & 0.1299 & 0.1316 & 0.1000 & 0.0682 & 0.1160 & 0.0468 & 0.0893 \\
\hline$P g d h$ & 143 & - & - & - & - & - & 0.0600 & $\begin{array}{c}0.0116 \\
(0.016)^{2}\end{array}$ \\
\hline \multirow[t]{2}{*}{ Pgm-1 } & 150 & - & 0.0263 & 0.0250 & 0 & 0.0072 & 0.0150 & 0.0084 \\
\hline & 170 & - & - & - & - & - & 0.0084 & 0.0184 \\
\hline \multirow[t]{3}{*}{ Pgm-2 } & 60 & 0.0974 & 0.0789 & 0.2250 & 0.0227 & 0.1014 & 0.0100 & 0.0083 \\
\hline & 150 & - & - & - & - & - & - & 0.0083 \\
\hline & 210 & - & - & - & - & - & - & 0.0050 \\
\hline
\end{tabular}

${ }^{1}$ Data from Romagnano, 1987.

${ }^{2}$ Data from Ross, 1983. 
with a mean value of $0.0226 \pm 0.1105$ (Table 2). Values were neither consistently negative nor positive, and the mean value is not significantly different from zero. This implies near-random mating within populations, and is generally supported by the analysis of Hardy-Weinberg equilibrium.

Calculation of Wright's $F_{\text {ST }}$ statistic based on all variable loci revealed no geographical population structure $\left(F_{\mathrm{ST}}=0.0067 \pm 0.0157\right.$, not significantly different from zero). Only the Pgm-2 locus showed a significant $(P<0.025)$ level of differentiation.

\section{Discussion}

\section{Population structure in North American starlings}

In general, allozyme studies of avian species suggest low levels of population structure presumably because of high levels of dispersal (see Barrowclough, 1980). In areas of their native range, starlings have a slight but statistically significant level of population subdivision $\left(F_{\mathrm{ST}}=0.0101 \pm 0.0017\right)$; introduced starlings in New Zealand exhibit a greater degree of subdivision $\left(F_{\mathrm{ST}}=0.0316 \pm 0.0103\right.$; Ross, 1983).

This study revealed no significant population subdivision. This may result from high dispersal rates in North American starlings, similarity of populations because of recent common ancestry, or both.

\section{Heterozygosity}

No geographical differentiation among localities was detected using Wright's $F_{\mathrm{ST}}$. For this reason, all individuals were pooled for analyses of heterozygosity and allelic diversity.
Measures of heterozygosity are commonly used to indicate levels of genetic variation within populations. The average level of observed heterozygosity in North America $(\approx 0.03)$ is lower than for many bird species, but still within the range of reported values for other genera of starlings and avian taxa in general (see Corbin, 1983).

Nei et al. (1975) examined the effects of population bottlenecks on two measures of genetic variation, heterozygosity and number of alleles. Although this treatment was based on an infinite alleles model that may or may not be applicable to electrophoretic data, the results are equivalent when other mutational models are considered (Chakraborty \& Nei, 1977).

In general, heterozygosity is decreased by population bottlenecks only if the bottleneck is extremely small. This is a function of the amount of heterozygosity represented in a sample of a given size:

$H_{\mathrm{S}}=H_{\mathrm{O}}\left(1-\frac{1}{2 N_{\mathrm{S}}}\right)$

where $H_{\mathrm{S}}$ is the expected heterozygosity of the sample, $H_{\mathrm{O}}$ is the heterozygosity of the original population, and $N_{\mathrm{S}}$ is the number of individuals in the sample. Thus, two individuals are expected to contain about $75 \%$ of the original heterozygosity of the sampled population.

This simplistic approach to understanding bottlenecks has important limitations (Nei et al., 1975). To test this prediction, one would have to sample the actual founding group. In addition, the loss of heterozygosity is exacerbated during the time period when the population is relatively small. Each successive generation is small compared to the original or

Table 2 Heterozygosities and Wright's $F$-statistics for starlings in North

America. Significance of $F_{\text {IS }}$ tested against the null hypothesis: $F_{\text {IS }}=0$,

$\chi^{2}=N\left(F_{\mathrm{IS}}^{2}\right)$ where $N$ is the number of individuals sampled, d.f. $=1$ (Nei, 1977);

significance of $F_{\mathrm{ST}}$ tested against the null hypothesis: $F_{\mathrm{ST}}=0, \chi^{2}=2 N F_{\mathrm{ST}}$,

d.f. $=(1-$ number of populations sampled $)=5($ Neel \& Ward, 1972)

\begin{tabular}{lccc}
\hline Locus $(N)$ & $H_{\mathrm{o}} \pm \mathrm{SE}$ & $F_{\mathrm{IS}} \pm \mathrm{SE}$ & $F_{\mathrm{ST}} \pm \mathrm{SE}$ \\
\hline Est-1 (136) & $0.1175 \pm 0.0126$ & $-0.0309 \pm 0.0016$ & 0 \\
Est-2 (98) & $0.0955 \pm 0.0430$ & $0.2416 \pm 0.2529^{*}$ & 0 \\
Pep- $A$ (138) & $0.1326 \pm 0.0128$ & $-0.0125 \pm 0.0323$ & 0 \\
Pep-C (138) & $0.2098 \pm 0.0271$ & $0.0055 \pm 0.1091$ & 0 \\
Pgm-1 (138) & $0.0257 \pm 0.0148$ & $-0.0002 \pm 0.0002$ & 0 \\
Pgm-2 (138) & $0.1823 \pm 0.0650$ & $-0.0677 \pm 0.0669$ & $0.0400^{*}$ \\
Mean over & $0.1272 \pm 0.0375$ & $0.0226 \pm 0.1105$ & $0.0067 \pm 0.0157$ \\
polymorphic loci (6) & & & \\
\hline
\end{tabular}

${ }^{*} P<0.05$. 
source population, and the cumulative effect is one of a series of bottlenecks. Eventually, the population becomes large enough to prevent any further loss of heterozygosity, and in fact begins to increase in heterozygosity with the addition of new alleles through mutation. This increase in heterozygosity is extremely slow. In generations, the time to reach a new equilibrium is $\approx 2 N /(4 N v+1)$ where $N$ is the total population size and $v$ is the mutation rate (Chakraborty \& Nei, 1977). For starlings this may be greater than 500000 generations. Thus, a population which has undergone a bottleneck will show a relatively rapid decrease in heterozygosity to some minimum value, and then an extremely slow increase until a new equilibrium is reached. For this study, the minimum expected heterozygosity is the most valid theoretical comparison: the population is too large to continue to lose heterozygosity, and insufficient time has passed for the population to have significantly increased in heterozygosity.

If the number of founders and the population growth rate are known, the minimum expected heterozygosity $\left(H_{\mathrm{m}}\right)$ relative to the original heterozygosity $\left(H_{\mathrm{o}}\right)$ can be calculated from

$\frac{H_{\mathrm{m}}}{H_{\mathrm{o}}}=\mathrm{e}^{\left\{\left(-\frac{1}{2 N_{\mathrm{O}}}\right)\left(\frac{w}{w-1}\right)\left[1+\frac{w}{4 N_{\mathrm{O}}(w+1)}\right]\right\}}$

where $N_{\mathrm{o}}$ is the number of founders and $w$ is the standard discrete population growth constant, $\lambda$ (Nei et al., 1975). For European Starlings, $\lambda$ can be estimated from demographic data (see Cabe, 1994). Life tables were constructed based on data from Kessel (1957); her data are consistent with other studies, and are the most extensive for North America (see Cabe, 1993). Estimates for $\lambda$ from her data range from 1.10-1.628. Feare (1984) estimated that there were $\approx 200$ million starlings in North America; this value could be reached in only 30 generations with the higher growth rate, which implies that it is an overestimate. About 150 generations are needed with the lower growth rate, which is thus an underestimate. The true growth potential must fall somewhere between these values.

Based on these growth rate estimates, the minimum expected heterozygosity can be calculated using eqn (2) above. With the estimates of $\lambda$ of 1.628 and 1.100 , the minimum expected heterozygosity is $98.7 \%$ and $94.7 \%$, respectively, of the heterozygosity of the original population.

Thus, the introduced population in North America is expected to show approximately the same level of heterozygosity as populations in Great Britain. This is consistent with the level of heterozy- gosity found in this study (uncorrected value $0.0311 \pm 0.0098$, see above) compared to comparable values calculated for Great Britain (uncorrected value $0.031 \pm 0.001$; Ross, 1983).

\section{Allelic diversity}

Nei et al. (1975) also examined the effect of founding bottlenecks on the number of alleles present in the new population relative to the original population. They used Monte Carlo simulation rather than analytical approaches which have been developed subsequently (Watterson, 1984), but require information which is not generally available and make assumptions which may not be met for most empirical data. In practice, the simplest analysis remains stochastic simulation.

Nei et al. (1975) used accepted assumptions of neutral allele distributions, and then randomly chose $2 N$ alleles to form a founder group of size $N$, and repeated this for varying numbers of generations. This neglects population growth subsequent to founding. In addition, they modelled populations that originally had an expectation of either 3.6 or 6.2 alleles per locus, a much higher number than reported for starlings. Their results suggested that even with large founding groups (100-1000) the proportion of alleles lost was substantial, in the range of $40-60 \%$. Populations which started with a greater number of alleles per locus lost more through the bottleneck, because a greater number of rare alleles were eliminated through drift; this finding is consistent with Sirkkomma (1983). Mutations in the generations immediately subsequent to the bottleneck are expected to have little effect if the product of the bottleneck size and mutation rate is much less than one, as is likely for most mutation rates (Maruyama \& Fuerst, 1985).

Ross (1983) reported 31 alleles for 11 variable loci in Great Britain; this study found 18 alleles from the same 11 loci (five of the loci were monomorphic). These electrophoretic data are in general agreement with the data of Evans (1980) from Great Britain and with the survey of 30 North American starlings by Romagnano (1987).

If the present study is compared directly to Ross (1983), whose protocols were followed, there is a loss in North America of $42 \%$ of the alleles at variable loci. This comparison may be somewhat misleading. Some 'missing' alleles in North America were probably missed by this study because of sampling error: this study analysed a maximum of 138 individuals compared with 298 in Ross's (1983) study (actual sample sizes were 74 for $M p i, 79$ for 
Pgdh, 108 for $I d h$ and $M e$, and 116 for $L d h$; sample sizes for polymorphic loci appear in Table 2). Although this would seem to make the comparison suspect, Maruyama \& Fuerst (1985, p. 680) concluded from mathematical analyses of bottlenecks that 'small samples taken from the population following reduction will contain almost all of the allelic variation that remains in the population. Increasing the sample size will therefore increase the number of sampled alleles only slightly.' This can be intuitively understood by noting that in an original group of $N$ individuals, the minimum possible allelic frequency is $1 /(2 N)$, and that the frequencies of any alleles persisting for more than a few generations after the founding event are likely to be somewhat higher than this minimum value (Cabe, 1994).

In general, these data are consistent with the theoretical findings of Nei et al. (1975), that rare alleles are lost even during large bottlenecks.

\section{Population bottlenecks: other empirical studies}

Many empirical studies have attempted to relate observed levels of genetic variation to presumed population history. For example, studies of introduced and other bottlenecked populations (Taylor \& Gorman, 1975; Huettel et al., 1980; Bryant et al., 1981; Parkin \& Cole, 1985; Woodruff et al., 1985; Johnson, 1988) are usually consistent with a population bottleneck during founding, although in most cases pertinent details about the founding event and original source population are lacking (but see Taylor \& Gorman, 1975). In these populations, a loss of alleles (especially rare ones) is often observed, although a decrease in heterozygosity is rarely reported (but see Johnson, 1988). These results are roughly consistent with theoretical predictions.

Packer et al. (1991) reported a significant decrease in heterozygosity in a lion population which underwent a severe bottleneck (15 individuals). In addition, the founders may have been related, and the reproductive success of individuals since the bottleneck may have shown high individual variance, causing additional loss of genetic variability. A loss of alleles is also suggested by their data.

McCommas \& Bryant's (1990) laboratory study of houseflies also provides excellent support for theoretical predictions of bottleneck effects. Leberg (1992) explicitly tested predictions about the loss of heterozygosity and allelic variation using replicate populations of mosquitofish in controlled conditions. His data offer strong support to the contention that a decrease in heterozygosity is expected only in cases where the bottleneck is extreme and prolonged.

\section{Conclusions}

The intentional introduction of the European starling to North America has provided an excellent natural experiment to test predictions about the genetic effect of population bottlenecks. It differs from other studies of the effects of bottlenecks in that the population restriction (about 100 individuals) was more moderate. Following the introduction, the growth rate and range expansion of the starling has been phenomenal.

Measures of heterozygosity in the introduced North American populations are indistinguishable from the level of heterozygosity in starlings in their native range in Great Britain. This is completely consistent with theoretical predictions; a decrease in heterozygosity is expected only if the bottleneck is extremely small or prolonged.

The only possible remaining signature of the founding bottleneck in starlings may be a decrease in allelic diversity. Alleles that were rare in the source population are at the greatest risk of being lost through the bottleneck. It is also likely that some rare alleles which were included in the original group of founders were lost in the first few generations subsequent to the introduction. If some loci have only one or two rare alleles, this may reduce the number of polymorphic loci.

Taken together, the heterozygosity and allelic diversity measures are in good agreement with predictions of the consequences of population bottlenecks based solely on theoretical considerations. The theoretical predictions are robust for allozyme data, and may apply to a wide variety of conditions (McCommas \& Bryant, 1990; Leberg, 1992). This suggests that if a bottleneck occurs, reasonable predictions about its genetic consequences can be made. Unfortunately, the converse is not true. Interpreting population history from genetic data is tenable only if information about the original source population is available.

\section{Acknowledgements}

I thank L. A. Beavers and D. N. Alstad for help in collecting. K. Corbin provided numerous helpful suggestions, in addition to valuable guidance and laboratory support for the entire study. K. Corbin, D. Alstad, J. Curtsinger and two anonymous reviewers gave many helpful comments. This work was 
supported by grants from the Dayton and Wilkie Funds (University of Minnesota), Sigma Xi, and an NSF doctoral fellowship.

\section{References}

BARRowClough, G. 1980. Gene flow, effective population sizes, and genetic variance components in birds. Evolution, 34, 789-798.

BRYANT, E. H., DIK, H. AND VAN DELDEN, w. 1981. Genetic variability in the face fly Musca autumnalis De Geer in relation to a population bottleneck. Evolution, 35, 872-881.

CABE, P. R. 1993. European Starling (Sturnus vulgaris). In: Poole, A. and Gill, F. (eds) The Birds of North America, no. 48. The Academy of Natural Sciences, Philadelphia; The American Ornithologist's Union, Washington, DC.

CABE, P. R. 1994. The Population Genetics of Introduced Species: the European Starling (Sturnus vulgaris) in North America. Ph.D. Thesis, University of Minnesota, St. Paul.

CHAKRABORTY, R. AND NEI, M. 1977. Bottleneck effects on average heterozygosity and genetic distance with the stepwise mutation model. Evolution, 31, 347-356.

CORBIN, K. w. 1983. Genetic structure and avian systematics. In: Johnston, R. F. (ed.) Current Ornithology, vol. 1, pp. 211-244. Plenum Press, New York.

CORBIN, K. W. AND WILKIE, P. J. 1988. Genetic similarities between subspecies of the white-crowned sparrow. Condor, 90, 637-647.

EVAns, P. G. H. 1980. Population Genetics of the European Starling (Sturnus vulgaris). Ph.D. Thesis, University of Oxford.

FEARE, C. J. 1984. The Starling. Oxford University Press, Oxford.

HUETTEL, M. P., FUERST, P. A., MARUYAMA, T. AND CHAKRABORTY, R. 1980. Genetic effects of multiple bottlenecks in the Mediterranean fruit fly (Ceratitis capitata). Genetics, 94, s47-s48.

JOHNSON, M. S. 1988. Founder effects and geographic variation in the land snail Theba pisana. Heredity, 61, $133-142$.

KESSEL, B. 1957. A study of the breeding biology of the European starling (Sturnus vulgaris L.) in North America. Am. Midl. Nat., 58, 257-231.

LEBERG, P. L. 1992. Effects of population bottlenecks on genetic diversity as measured by allozyme electrophoresis. Evolution, 46, 477-494.

McCOMMAS, S. A. AND BRYANT, E. H. 1990. Loss of electrophoretic variation in serially bottlenecked populations. Heredity, 64, 315-321.

MARUYAMA, T. AND FUERST, P. 1985. Population bottlenecks and nonequilibrium models in population genet- ics. II. Number of alleles in a small population that was formed by a recent bottleneck. Genetics, 111, 675-689.

NEEL, J. V. AND WARD, R. H. 1972. The genetic structure of a tribal population, the Yanomama Indians. VI. Analysis by $F$-statistics including a comparison with the Makiritare and Xavante. Genetics, 72, 636-666.

NEI, M. 1977. F-statistics and the analysis of gene diversity in subdivided populations. Ann. Hum. Genet., 41, $225-233$.

NEI, M. 1978. Estimation of average heterozygosity and genetic distance from a small number of individuals. Genetics, 89, 583-590.

NEI, M., MARUYAMA, T. AND CHAKRABORTY, R. 1975. The bottleneck effect and genetic variability in populations. Evolution, 29, 1-10.

O'BRIEN, S. J., WILDT, D. E., BUSH, M., CARO, T. M., FITZGIBBON, C., AGGUNDEY, I. AND LEAKEY, R. 1987. East African cheetahs: Evidence for two population bottlenecks? Proc. Natl. Acad. Sci. U.S.A., 84, 508-511.

PACKER, C., PUSEY, A., ROWLEY, H., GILBERT, D. A., MARTENSON, J. AND O'BRIEN, S. J. 1991. Case study of a population bottleneck: Lions of the Ngorongora Crater. Conserv. Biol, 5, 219-237.

PARKIN, D. T. AND COLE, S. R. 1985. Genetic differentiation and rates of evolution in some introduced populations of the House Sparrow, Passer domesticus, in Australia and New Zealand. Heredity, 54, 15-23.

romagnano, L. C. 1987. Intraspecific Brood Parasitism in the European Starling (Sturnus vulgaris). Ph.D. Thesis, Rutgers University, New Brunswick, NJ.

ROMAGNANO, L., HOFFENBERG, A. S. AND POWER, H. W. 1990. Intraspecific brood parasitism in the European starling. Wilson Bulletin, 102, 279-291.

Ross, H. A. 1983. Genetic differentiation of starling (Sturnus vulgaris: Aves) populations in New Zealand and Great Britain. J. Zool. Lond., 201, 351-362.

SiRккомма, s. 1983. Calculations on the decrease of genetic variation due to the founder effect. Hereditas, 99, 11-20.

TAYLOR, C. E. AND GORMAN, G. C. 1975. Population genetics of a 'colonizing' lizard: natural selection for allozyme morphs in Anolis grahami. Heredity, 35, 241-247.

WAtterson, G. A. 1984. Allele frequencies after a bottleneck. Theor. Pop. Biol, 26, 387-407.

WAYNe, R. K., GILBERT, D. A., LEHMAN, N., HANSEN, K., EISENHOWER, A., GIRMAN, D. ET AL. 1991. Conservation genetics of the endangered Isle Royale gray wolf. Conserv. Biol., 5, 41-51.

WoOdrufF, D. S., Mulvey, M. AND. YIPP, w. 1985. Population genetics of Biomphalaria straminea in Hong Kong. J. Hered., 76, 355-360.

Wright, s. 1978. Evolution and the Genetics of Populations, vol. 4, Variability Within and Among Natural Populations. The University of Chicago Press, Chicago. 